Max-Planck-Institut für demografische Forschung

Max Planck Institute for Demographic Research

Doberaner Strasse $114 \cdot$ D-18057 Rostock $\cdot$ GERMANY

Tel +49 (0) 3812081 - 0; Fax +49 (0) 3812081 - 202;

http://www.demogr.mpg.de

MPIDR WORKING PAPER WP 2002-021

MAY 2002

\title{
Eine Mehrebenenanalyse regionaler Einflüsse auf die Familiengründung westdeutscher Frauen in den Jahren 1984 bis 1999
}

Karsten Hank (hank@demogr.mpg.de)

This working paper has been approved for release by: Hans-Peter Kohler (kohler@demogr.mpg.de) Head of the Research Group on Social Dynamics and Fertility.

(C) Copyright is held by the authors.

Working papers of the Max Planck Institute for Demographic Research receive only limited review. Views or opinions expressed in working papers are attributable to the authors and do not necessarily reflect those of the Institute. 


\title{
Eine Mehrebenenanalyse regionaler Einflüsse auf die Familiengründung westdeutscher Frauen in den Jahren 1984 bis 1999
}

\author{
Karsten Hank, Max-Planck-Institut für demografische Forschung, Rostock ${ }^{\star}$
}

Zusammenfassung: Der Artikel untersucht die Bedeutung regionaler Einflussfaktoren auf der Kreisebene für den Prozess der Familiengründung westdeutscher Frauen in den 1980er und 1990er Jahren. Als theoretischer Rahmen wird ein Mehrebenenmodell soziologischer Erklärung vorgeschlagen. Die empirische Analyse des Übergangs zur ersten Ehe sowie der Geburt des ersten und zweiten Kindes erfolgt auf der Grundlage von Mikrodaten des Sozio-oekonomischen Panels (SOEP) und unter Verwendung eines Methoden-Mixes aus ereignisanalytischen und mehrebenenanalytischen Verfahren. Die beiden wichtigsten Befunde sind erstens, dass regionale Unterschiede im Geburtenverhalten weitestgehend durch Kontrolle individueller Merkmale - vor allem Familienstand und Alter - erklärt werden können, und zweitens, dass es eine signifikante regionale Varianz im Heiratsverhalten gibt, die weder auf Kompositionseffekte noch auf strukturelle Kontexteffekte zurückzuführen ist. Regionale Einflüsse auf Fertilitätsentscheidungen westdeutscher Frauen haben demnach keine eigenständige Qualität, sondern werden über einen latenten Kontexteffekt raumgebundener sozio-kultureller Milieus auf die Wahrscheinlichkeit einer Eheschließung lediglich indirekt vermittelt.

* Korrespondenzanschrift: Max-Planck-Institut für demografische Forschung, Doberaner Str. 114, 18057 Rostock. Email: hank@ demogr.mpg.de.

Für hilfreiche Anregungen und kritische Hinweise danke ich Johannes Huinink und Hans-Peter Kohler. Die multivariaten Modelle wurden während eines Gastaufenthaltes bei der SOEPGruppe am DIW Berlin geschätzt.

Die hier vertretenen Ansichten sind die des Autors und müssen nicht notwendigerweise denen des Max-Planck-Instituts für demografische Forschung entsprechen. 


\section{Einleitung und Hintergrund der Untersuchung}

Vor, während und auch nach dem demografischen Übergang des ausgehenden 19. und beginnenden 20. Jahrhunderts war Deutschland durch ein im Vergleich zu anderen europäischen Ländern überdurchschnittlich hohes Maß an regionaler Heterogenität hinsichtlich verschiedenster demografischer Merkmale gekennzeichnet (vgl. Knodel 1974; Knodel 1988). Die Unterschiede in der Sterblichkeit oder im reproduktiven Verhalten der Bevölkerung in den einzelnen Landesteilen korrespondierten dabei deutlich mit der politischen und kulturellen Fragmentierung des Reiches, das erst ab 1870 eine staatliche Einheit bildete.

In der Folge des sogenannten zweiten demografischen Übergangs - und dem damit einhergehenden massiven Geburtenrückgang ab Mitte der 60er Jahre des vorigen Jahrhunderts - wuchs in den 1980er Jahren erneut das Interesse an der Untersuchung regionaler Fertilitätsunterschiede (z.B. Akademie für Raumforschung und Landesplanung 1983; Bähr und Gans 1991). Mit der Wiedervereinigung der beiden deutschen Staaten gerieten jedoch relativ kleinräumige regionale Unterschiede innerhalb West- und Ostdeutschlands zugunsten räumlich wenig differenzierter Vergleiche der demografischen Lage in Ost und West weitgehend in den Hintergrund des wissenschaftlichen Interesses (z.B. Eberstadt 1994; Conrad et al. 1996). ${ }^{1}$

Während die bereits $\mathrm{zu}$ DDR-Zeiten relativ kleinen regionalen Fertilitätsunterschiede in Ostdeutschland nach der Wiedervereinigung noch weiter abgenommen haben (z.B. Gans 1996), bestätigt eine Analyse aktueller Regionaldaten

\footnotetext{
${ }^{1}$ Erwähnenswerte Ausnahmen stellen die familiensoziologischen Arbeiten von Bertram und Nauck dar (z.B. Bertram 1996; Bertram et al. 2000; Nauck 1995).
} 
der amtlichen Statistik jedoch die fortdauernde Existenz deutlicher Fertilitätsunterschiede zwischen westdeutschen Kreisen und eine bemerkenswerte Persistenz räumlicher Muster (vgl. hierzu ausführlich Hank 2001). Dabei ist zunächst festzuhalten, dass sich die Fertilitätsniveaus in städtischen und ländlichen Regionen auch in der jüngeren Vergangenheit nicht angeglichen haben. Weiterhin kann festgestellt werden, dass der Abstand zwischen den höchsten Geburtenraten in einigen Teilen Nordwest- und Süddeutschlands einerseits, und den niedrigsten Werten in einer Reihe von Universitätsstädten andererseits, größer ist, als die derzeit beobachteten Unterschiede zwischen den alten und neuen Bundesländern. So konnte Mitte der 1990er Jahre in Heidelberg eine allgemeine Geburtenziffer von nur 0.9 beobachtet werden, während der Landkreis Cloppenburg mit einem Wert von 1.9 fast das zur Bestandserhaltung notwendige Fertilitätsniveau erreichte.

Weitere deskriptive Befunde deuten auf einen möglichen Zusammenhang zwischen dem allgemeinen Fertilitätsniveau eines Kreises und strukturellen Merkmalen, wie der Bevölkerungsdichte, der Migration insbesondere junger Familien, und der regionalen Arbeitsmarkt- bzw. Berufsstruktur hin (Hank 2001: 251ff.). Es wäre jedoch verfehlt, auf der Grundlage ökologischer Korrelationen Aussagen über ursächliche Zusammenhänge treffen $\mathrm{zu}$ wollen. Dafür bedarf es vielmehr eines einheitlichen theoretischen wie empirischen Rahmens, der es ermöglicht, jene Mechanismen zu identifizieren und in ihrer Wirkungsweise $\mathrm{zu}$ analysieren, welche die eigentliche Verknüpfung zwischen Kontextmerkmalen und individuellen Entscheidungen herstellen. Ein geeignetes Instrument hierfür stellt die Mehrebenenanalyse dar (z.B. Courgeau und Baccaini 1998; DiPrete und Forristal 1994). 
Entscheidungen hinsichtlich der Familiengründung und die daraus folgenden Konsequenzen werden in dieser Untersuchung aus einer Mehrebenenperspektive heraus als Ereignisse im Lebensverlauf verstanden, die sowohl durch individuelle Eigenschaften als auch durch die spezifischen Lebensbedingungen im sozialräumlichen Kontext der Handelnden determiniert werden. Die hier vertretene Argumentationslinie folgt damit theoretisch weitgehend dem von Esser (1988; 1996) und Coleman (1986; 1990) vorgeschlagenen Modell soziologischer Erklärung.

Empirische Analysen, die das Verhältnis zwischen regionalen Opportunitätsstrukturen, sozialen Interaktionsprozessen, und kulturellen Milieus auf lokaler Ebene, sowie reproduktivem Verhalten auf der Individualebene untersuchen, sind bislang - zumindest für den europäischen Raum - eher selten. ${ }^{2}$ Die wenigen für Deutschland vorliegenden Untersuchungen berücksichtigen lediglich einzelne Aspekte der regionalen Infrastruktur, wie z.B. die Versorgung mit Kinderbetreuung (Hank und Kreyenfeld 2002, im Druck), basieren auf nicht-repräsentativen Stichproben räumlicher Kontexte (z.B. Kopp 2000), oder beziehen sich auf relativ große Gebietseinheiten, wie z.B. Raumordnungsregionen (Hank 2002, im Druck). Diese Studie soll dazu beitragen, bestehende Lücken zu füllen. Dazu gehört auch die bislang fehlende Einbeziehung des Heiratsverhaltens. Konkret wird nach der Bedeutung verschiedener regionaler Einflussfaktoren auf der Kreisebene für den Prozess der Familiengründung westdeutscher Frauen in den 1980er und 1990er Jahren gefragt. Die Analyse des Übergangs zur ersten Ehe sowie der Geburt des ersten und zweiten Kindes erfolgt auf der Grundlage von Mikrodaten des Sozio-oekonomischen Panels (SOEP) und unter

\footnotetext{
${ }^{2}$ In der nordamerikanischen Literatur findet sich hingegen eine rasch wachsende Zahl von Studien, die den Einfluss von Nachbarschaftseffekten auf familiäre Ereignisse untersuchen (z.B. Billy und Moore 1992; South and Crowder 1999; Teachman and Crowder 2002).
} 
Verwendung eines Methoden-Mixes aus ereignisanalytischen und mehrebenenanalytischen Verfahren.

\section{Theoretisches Modell und Hypothesen}

\section{$\underline{2.1 \text { Ein Mehrebenen-Ansatz zur Erklärung sozialer Phänomene }}$}

Das Verhältnis von Individuum und Kontext ist bis zum heutigen Tag eines der wichtigsten und am meisten diskutierten Themen in den Sozialwissenschaften (z.B. Alexander et al. 1987). In diesem Zusammenhang stellt der hier vorgeschlagene Mehrebenenansatz einen wichtigen Beitrag zur Überwindung des in der Vergangenheit oft ausgeprägten Mikro-Makro-Dualismus in der Sozialforschung dar (z.B. Huber 1991). Dabei ist es wichtig, sich die Mikrofundierung des Mehrebenenansatzes bewußt zu machen: während individuelle Handlungsspielräume einerseits durch gegebene ökonomische, soziale und kulturelle Rahmenbedingungen determiniert sind, werden eben diese kontextuellen Rahmenbedingungen gleichzeitig durch das Verhalten individueller Akteure beeinflußt. Gesellschaftliche Dynamik ist also das Produkt der dialektischen Beziehung zwischen individuellem Handeln und Sozialstruktur (z.B. Erbring und Young 1979; Hernes 1976).

Folgt man Siegwart Lindenberg (1990: 736), bezieht sich der analytische Primat der Sozialwissenschaften auf die gesellschaftliche Makroebene, während ihr theoretischer Primat auf der Mikroebene liegt. D.h., makrosoziologische Phänomene sind der eigentlich interessierende Gegenstand der Disziplin, der aber mikrosoziologisch über individuelles Handeln und die Interaktion zwischen Individuen erklärt werden soll. Eine solche akteursbezogene Perspektive impliziert, dass jede Erklärung sozialer 
Prozesse drei grundlegende analytische Schritte beinhalten muss (vgl. Coleman 1990: 8; Esser 1996: 94ff.):

\begin{abstract}
Abbildung 1
(1) Zunächst müssen die Determinanten der individuellen Handlungssituation bestimmt werden. Die Logik der Situation wird dabei durch den individuellen Hintergrund der Handelnden und den sozialen Kontext strukturiert.

Der Kontext bildet den Bezugsrahmen für die Aktionen und Interaktionen von Individuen und Gruppen in einer spezifischen Situation (vgl. Münch und Smelser 1987: 381f.). In diesem Sinne können regionale soziale Kontexte - um die es hier geht - als im Wesentlichen aus drei Komponenten bestehend konzipiert werden: soziale Bezugsrahmen (z.B. Normen), kulturelle Bezugsrahmen (z.B. Werte) und Opportunitätsstrukturen (z.B. Infrastruktur). Jede dieser drei Komponenten ist durch spezifische kollektive Eigenschaften charakterisiert, die zu kontextuellen Eigenschaften der Individuen werden (vgl. Lazarsfeld und Menzel 1969), d.h. zu relativ invarianten strukturellen Verhaltensparametern.
\end{abstract}

Der individuelle Hintergrund besteht im Wesentlichen aus zwei Dimensionen: Erstens hat jedes Individuum (mehr oder weniger) Zugang zu verschiedenen Ressourcen wie ökonomischem, sozialem oder kulturellem Kapital. Zweitens ist die psycho-soziale Disposition, oder Identität, der Handelnden zu berücksichtigen. Sie umfaßt Elemente wie Werte oder Erwartungen, und schließlich die Präferenzstruktur des Individuums.

Sogenannte Brückenhypothesen (vgl. Esser 1998) verknüpfen schließlich die objektiven Möglichkeiten und Grenzen einer spezifischen Situation mit den jeweiligen individuellen Erwartungen und der subjektiven Interpretation und Bewertung dieser Situation. Ohne Brückenhypothesen wäre es nicht möglich, individuelle Entscheidungen 
zu verstehen, da die konkreten Randbedingungen der Handlungstheorie undefiniert blieben.

(2) Das Handlungsmodell bestimmt die Logik der Selektion. Die Handlungstheorie bildet damit den nomologischen Kern jeder soziologischen Erklärung, d.h. sie stellt den kausalen Zusammenhang zwischen individuellen Eigenschaften und der Wahl einer bestimmten Handlungsalternative her. Das handlungstheoretische Modell dieser Untersuchung entspricht dem u.a. von Lindenberg (1990) vorgeschlagenen Konzept des homo socio-oeconomicus, welches auf einem flexiblen rational-choice Ansatz basiert (vgl. auch Hechter und Kanazawa 1997: 196f.).

(3) Schließlich muss die Logik der Aggregation geklärt werden, also die Transformationsregeln, nach denen aus den z.T. unintendierten Folgen individueller Handlungen kollektive soziale Konsequenzen entstehen (z.B. Lindenberg 1977). Üblicherweise ist das Transformationsproblem am schwierigsten zu lösen, doch bei der vorliegenden empirischen Fragestellung genügt i.d.R. ein einfacher Aggregationsmechanismus, um von individuellen Entscheidungen hinsichtlich der Familiengründung zu den entsprechenden demografischen Maßzahlen zu gelangen (z.B. Münch und Smelser 1987: 376f.).

\subsection{Makro-Mikro-Brücken: Wie regionale soziale Kontexte auf individuelle}

Entscheidungen zur Familiengründung wirken

Die Verwendung von Aggregatvariablen in empirischen Untersuchungen individueller Entscheidungsprozesse ist oft unter Hinweis auf allgemeine methodologische Probleme in Frage gestellt worden. Hauptargumente in dieser Diskussion sind die Behauptung, dass beobachtete Kontexteffekte häufig allein auf einer mangelnden Berücksichtigung 
relevanter Individualvariablen beruhen, und dass viele Studien die für den angenommenen Wirkungszusammenhang zwischen Eigenschaften des Kontextes und individuellem Verhalten ursächlichen sozialen Mechanismen nicht hinreichend darlegen (vgl. Hauser 1974).

Daher muss vor der empirischen Analyse geklärt werden, was wichtige Kontrollvariablen für den individuellen Hintergrund der Handelnden sind und welche Prozesse Kontexteffekte vermitteln. Nauck (1995: 95) weist z.B. auf die potenzielle Verhaltenswirksamkeit sozialer Kontexte „als 1) Opportunitätsstrutkuren für die Realisierung individueller Handlungspräferenzen, als 2) Orte sozialer Kontrolle oder 3) der Tradierung von Lebensformen und kulturellen Mustern, als 4) Ziele selektiver Migration und als 5) Objekte der Identifikation“ hin. Ich selbst schlage in Anlehnung hieran - und mit Blick auf die oben genannten Hauptkomponenten regionaler sozialer Kontexte - vor, zwischen Opportunitätsstrukturen, lokalen Interaktionsprozessen und kulturellen Milieus zu unterscheiden, die alle zu Parametern individuellen Verhaltens werden können (vgl. Hank 2002, im Druck).

Die folgenden Hypothesen beschreiben die wesentlichen Annahmen, die sich daraus für die Untersuchung des Zusammenhangs zwischen regionalen sozialen Kontexten und der Familiengründung westdeutscher Frauen in den 1980er und 1990er Jahren ergeben. Die Auswahl der relevanten Regionalvariablen erfolgt dabei z.T. mit Blick auf die Verfügbarkeit entsprechender Daten für eine spätere empirische Auswertung. Da sich die individuellen Kontrollvariablen bereits in früheren Untersuchungen oft als relevant für das Geburten- und Heiratsverhalten herausgestellt haben, werden sie hier nicht ausführlicher diskutiert. 


\section{Individualmerkmale}

Das Alter ist die wichtigste bio-soziale Determinante der Stellung des Individuums im Lebensverlauf. Dabei wird ein nicht-monotoner Effekt des Alters auf die Familiengründung angenommen, d.h. die Wahrscheinlichkeit einer ersten oder weiteren Mutterschaft bzw. Ehe sollte zunächst mit dem Alter steigen, um in späteren Lebensphasen wieder zu sinken.

Der Einfluss individueller Humankapitalinvestitionen auf den Prozess der Familiengründung ist sowohl aus ökonomischer (z.B. Becker 1993: Kapitel 5) wie auch aus soziologischer Perspektive (z.B. Blossfeld und Huinink 1991) umfassend analysiert worden. Einerseits gewinnen besser ausgebildete Frauen durch ihre höheren Bildungsinvestitionen an ökonomischer Unabhängigkeit von einem potenziellen Partner, bei gleichzeitig steigenden Opportunitätskosten der Kindererziehung. Beides sollte mindestens zu einer Verschiebung der Familiengründung führen. Andererseits erhöht Bildung die eigene Attraktivität auf dem Heiratsmarkt und das zum Unterhalt einer Familie notwendige wirtschaftliche Potenzial. Der Nettoeffekt eines höheren individuellen Bildungsniveaus auf die Familiengründung ist daher nicht eindeutig vorhersagbar. In jedem Fall sollte das Risiko einer ersten Ehe und/oder Mutterschaft am geringsten sein, solange die Frau sich in Ausbildung befindet.

Schließlich sollten Verheiratete eine höhere Wahrscheinlichkeit haben Mutter zu werden, und Mütter sollten eine größere Neigung zur Eheschließung zeigen. Aufgrund einer mutmaßlich traditionelleren Wertorientierung sollten darüber hinaus Ausländerinnen ein höheres Familiengründungsrisiko aufweisen, als deutsche Frauen. 


\section{Regionale Kontextmerkmale}

Eine der wichtigsten Determinanten der Lebensbedingungen in einer Region sind lokale Opportunitätsstrukturen, die u.a. mit dem jeweiligen Urbanisierungsgrad variieren sollten. Städtische Räume bieten im Vergleich zu ländlichen Gegenden üblicherweise mehr Alternativen zur traditionellen Familiengründung und stellen gleichzeitig eine weniger vorteilhafte Umgebung für das Aufwachsen von Kindern dar. Daher wird angenommen, dass Frauen eine abnehmende Neigung $\mathrm{zu}$ Ehe und Elternschaft aufweisen, wenn die Bevölkerungsdichte ihres Wohnortes, bzw. Kreises, steigt (z.B. Hunink und Wagner 1989; Lichter et al. 1991).

Die Verfügbarkeit von Kinderbetreuung spielt eine entscheidende Rolle für die Vereinbarkeit von Mutterschaft und Erwerbstätigkeit der Frau, so dass die Möglichkeit außerhäuslicher Betreuung von Kindern zu einem zentralen Element der regionalen Opportunitätsstruktur wird. Da die Versorgung mit angemessenen Betreuungsmöglichkeiten die Opportunitätskosten der Kindererziehung für Frauen die am Erwerbsleben teilnehmen möchten reduziert, sollte die Wahrscheinlichkeit Kinder zu bekommen mit dem Angebot an öffentlicher Kinderbetreuung steigen (z.B. Kravdal 1996).

Mangel an Partnern auf dem Heiratsmarkt, z.B. wegen eines unausgeglichenen Geschlechterverhältnisses, führt häufig zu einem höheren Anteil Unverheirateter, oder zumindest $\mathrm{zu}$ einer zeitlichen Verzögerung von Eheschließungen. Es wird daher allgemein angenommen, dass Frauen, die einer großen Anzahl Männer auf dem lokalen Heiratsmarkt gegenüberstehen, hohe Heiratsraten aufweisen werden (z.B. South und Lloyd 1992). 
Beschäftigungsmöglichkeiten im Dienstleistungssektor werden als bedeutsam für die Karrierechancen von Frauen betrachtet (z.B. Blossfeld 1987). Da mit einer steigenden Zahl von Arbeitsplätzen im tertiären Sektor sowohl die Chancen auf wirtschaftliche Autonomie, als auch die Opportunitätskosten der Mutterschaft wachsen sollten, wird angenommen, dass in diesem Fall die Wahrscheinlichkeit einer Frau zu heiraten oder ein Kind zu bekommen sinkt.

Die Richtung eines möglichen Effekts der regionalen Arbeitslosenquote auf individuelle Fertilitätsentscheidungen ist schwer vorhersagbar (vgl. Kravdal 2002: Abschnitt 3). Einerseits kann mit Blick auf die kurzfristigen beruflichen Opportunitätskosten infolge einer Familiengründung angenommen werden, dass die Wahrscheinlichkeit ein Kind zu bekommen jeweils mit der Arbeitslosenquote steigen oder fallen sollte, da das Arbeitsangebot von Frauen auch von der Arbeitsnachfrage abhängt. Andererseits kann die Situation auf dem lokalen Arbeitsmarkt auch als Indikator für die allgemeine wirtschaftliche Lage einer Region betrachtet werden. Es könnte also erwartet werden, dass Frauen ein Kind eher dann bekommen werden, wenn die Arbeitslosigkeit sinkt, da die ökonomischen Kosten eines Kindes in einer gesamtwirtschaftlich positiveren Situation tragbarer erscheinen sollten (z.B. Hoem 2000: Abschnitt 5).

Die Wirkungsweise der regionalen Arbeitslosenquote auf das Heiratsverhalten ist ebenfalls nicht eindeutig zu prognostizieren. Wird die Arbeitsmarktlage als schwierig eingeschätzt, könnten Frauen in der Institution Ehe wirtschaftliche Sicherheit suchen, indem sie ihr Einkommen mit dem des Partners poolen. Gleichzeitig kann verbreitete Arbeitslosigkeit dazu führen, dass die Zahl der ökonomisch attraktiven Partner auf dem 
Heiratsmarkt abnimmt, was zu einem Aufschub von Eheschließungen führen könnte (z.B. Lichter et al. 1991).

Auch der Gesamteffekt der Frauenerwerbsquote auf die individuelle Entscheidung eine Familie zu gründen bleibt unklar (vgl. Brewster und Rindfuss [2000] für einen aktuellen Überblick). Die allgemeine Erwerbsbeteiligung von Frauen ist ein Indikator für das $\mathrm{Maß}$ an gesellschaftlichen Erwartungen und Möglichkeiten hinsichtlich ihrer ökonomischen Unabhängigkeit von einem männlichen Partner. Wenn man davon ausgeht, dass das Beispiel anderer Frauen eine Rolle für die individuelle Motivation zur Erwerbstätigkeit (und damit zum Aufschub oder Verzicht einer traditionellen Familiengründung) spielt, sollte die Neigung zu Mutterschaft und Ehe mit einer steigenden Frauenerwerbsquote zurückgehen. Gleichzeitig könnte jedoch gerade die zunehmende Partizipation von Frauen am Erwerbsleben dazu führen, dass die Bedürfnisse von Müttern auf dem Arbeitsmarkt stärker berücksichtigt werden. Also könnte die individuelle Wahrscheinlichkeit ein Kind zu bekommen mit einer höheren Erwerbsbeteiligung von Frauen sogar steigen.

Schließlich werden Akteure durch Verhaltenserwartungen und tatsächliches Verhalten in ihrer sozialen Umwelt beeinflusst. Dies kann u.a. zur Entstehung und Reproduktion von Normen, z.B. bezüglich des Alters und der Reihenfolge bei einzelnen Schritten der Familiengründung führen (z.B. Blossfeld und Huinink 1991; Settersten und Hägestad 1996). Da kulturell konservative, d.h. strukturerhaltende Kräfte einen stärkeren Einfluss in eher homogenen sozialen Kontexten haben sollten, kann dort ein höheres individuelles Familiengründungsrisiko vermutet werden, wo hohe Geburtenund Heiratsraten sowie eine dominierende Wertorientierung beobachtet werden. 
Letztere kann sowohl religiös als auch politisch begründet sein (z.B. Lesthaeghe und Surkyn 1988).

\section{Datenbasis und methodisches Vorgehen}

\subsection{Datenbasis}

Datenbasis für diese Studie ist das Sozio-oekonomische Panel des Deutschen Instituts für Wirtschaftsforschung (vgl. SOEP-Gruppe [2001] für einen Überblick). Dabei handelt es sich um eine seit 1984 (in Westdeutschland) jährlich durchgeführte Längsschnittuntersuchung privater Haushalte. Die vollständige Heirats- und Geburtsbiographie aller an der Befragung teilnehmenden Frauen ist im Datensatz enthalten. Die vorliegende Analyse bezieht sich auf die erste Eheschließung sowie die Geburt des ersten und zweiten Kindes bis zum Jahr 1999.

Die Individualdaten des SOEP können mit Informationen über die Kreise, in denen die Befragten leben, verknüpft werden. Leider sind die meisten interessierenden Regionalvariablen nur für zwei Zeitpunkte verfügbar. Die DJI Regionaldatenbank enthält regionale Indikatoren für die zweite Hälfte der 1980er Jahre, während die Statistik regional Datenbank der Statistischen Ämter des Bundes und der Länder entsprechende Informationen für die Mitte der 1990er Jahre bereitstellt. Im Hinblick auf zeitabhängige Regionalvariablen wird der Beobachtungszeitraum des Panels daher in zwei Hälften geteilt, nämlich von 1984 bis 1991 sowie von 1992 bis 1999. Tabelle 1 gibt einen Überblick über alle in der multivariaten Analyse verwendeten Individual- und Kontextvariablen.

\section{Tabelle 1}


Nur Befragte aus den beiden ursprünglichen SOEP-Stichproben, d.h. Westdeutsche sowie Griechinnen, Italienerinnen, Spanierinnen, Türkinnen und Frauen aus Ex-Jugoslawien die bereits 1984 in der Bundesrepublik gelebt haben, werden in die Analyse aufgenommen. Personen, die während des Untersuchungszeitraums innerhalb Westdeutschlands umziehen, werden verfolgt, so dass migrationsbedingte Veränderungen, z.B. der regionalen Opportunitätsstruktur, erfasst werden. ${ }^{3}$ Die untere Altersgrenze für die Analyse des Übergangs zur ersten Ehe und zum ersten Kind liegt bei 20, die obere Altersgrenze bei 35 Jahren. Die Analyse der Geburt des zweiten Kindes bezieht sich auf Frauen im Alter von mindestens 25 bis maximal 40 Jahren (vgl.

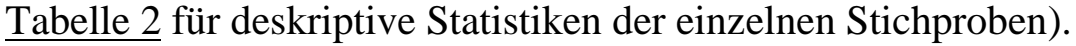

\section{Tabelle 2}

\subsection{Zeitdiskrete logistische Mehrebenenmodelle}

Diese Untersuchung verwendet zeitdiskrete Mehrebenenmodelle um das Risiko einer Frau zu schätzen, innerhalb eines Intervalls von einem Jahr ihr erstes oder zweites Kind zu bekommen, bzw. erstmals zu heiraten (vgl. Barber et al. [2000] für eine ausführlichere methodische Darstellung). Zeitdiskrete Hazardraten werden häufig als

\footnotetext{
${ }^{3}$ Hierbei ist allerdings zu berücksichtigen, dass unbeobachtete Individualmerkmale sowohl das reproduktive Verhalten als auch Wanderungsentscheidungen der Befragten beeinflussen können. Kontexteffekte könnten also durch selektive Migration verstärkt oder sogar nur vorgetäuscht werden (z.B. Manski 1993). Zwei gängige Methoden um dies statistisch zu kontrollieren sind die Verwendung von Instrumentenvariablen sowie die Schätzung von Modellen mit fixen Effekten. Beide Verfahren sind jedoch sehr voraussetzungsvoll und können in dieser Analyse nicht verwendet werden. Um die Robustheit der hier geschätzten Koeffizienten gegenüber möglichen Selektionseffekten dennoch überprüfen zu können, wurden alternativ alle multivariaten Modelle auch unter Ausschluß von Migrantinnen gerechnet. In keinem Fall haben sich dabei Hinweise auf das Vorliegen endogener Kontexteffekte ergeben.
} 
logistische Funktionen spezifiziert (z.B. Allison 1982; Yamaguchi 1991), wobei das zeitdiskrete Logitmodell den Effekt der Kovariaten auf den log-odds des Ereignisses schätzt. Klumpungen von Individuen in einem gemeinsamen Kontext verletzen jedoch die Standardannahme unabhängiger Fehlerterme. Dies kann dazu führen, dass für die Aggregatvariablen ineffiziente Parameter und nach unten verzerrte Standardfehler geschätzt werden. Logistische Mehrebenenmodelle berücksichtigen diese Problematik (vgl. Guo und Zhao [2000] für einen Überblick).

In der vorliegenden Untersuchung wird davon ausgegangen, dass der Effekt der erklärenden Variablen in allen Kontexten, d.h. Kreisen, gleich ist. Die Regressionskonstante kann jedoch mit dem jeweiligen Kontext variieren, d.h. sie besteht aus einer fixen und einer zufälligen Komponente. Dieses random intercept model (vgl. Snijders und Bosker 1999: Kapitel 4) nimmt im konkreten Fall folgende Form an:

$$
\log \left[p_{i j t} /\left(1-p_{i j t}\right)\right]=b_{0}+b_{1} x_{i j}+b_{2} z_{i j t}+b_{3} v_{j}+b_{4} w_{j t}+u_{0 j}
$$

wobei $p_{i j t}$ die Wahrscheinlichkeit angibt, mit der Individuum $i$ in Region $j$ das in Frage stehende Ereignis (d.h. erste oder zweite Geburt, bzw. erste Heirat) im Jahr $t$ erlebt. $x_{i j}$ und $v_{j}$ sind Vektoren zeitunabhängiger erklärender Variablen, während $z_{i j t}$ und $w_{j t}$ Vektoren zeitabhängiger erklärender Variablen im Jahr $t$ sind. Der Parameter $b_{0}$ bildet den fixen Teil der Regressionskonstante und der normalverteilte Fehlerterm $u_{0 j}$ stellt deren zufällige Komponente dar.

Dieser regionale Zufallskoeffizient $u_{0 j}$, der für alle Beobachtungen innerhalb eines Kreises den gleichen Wert annimmt, beschreibt die Abweichung jedes einzelnen Kontexts von $b_{0}$ (between-context variance). Damit wird nicht nur für die Korrelation zwischen Individuen innerhalb desselben Kontexts kontrolliert, sondern es werden auch 
sonst unbeobachtete regionale Effekte erfasst. Unterscheidet sich die Varianz von $u_{0 j}$ statistisch signifikant von 0, liegen solche Kontexteffekte vor.

In zeitdiskreten Logitmodellen stellt jede Periode in der ein Individuum beobachtet wird eine separate und unabhängige Beobachtung dar. Weil Geburten einer bestimmten Ordnung oder die erste Eheschließung einmalige Ereignisse sind, wird das Individuum nach Eintritt des Ereignisses von weiteren Beobachtungen ausgeschlossen. Die hieraus resultierende unzureichende Variation der abhängigen Variablen verhindert die Identifizierung unbeobachteter Individualeffekte, die in einem herkömmlichen Paneldatenmodell möglich und notwendig wäre.

\section{Regressionsergebnisse}

Die Ergebnisse der multivariaten Analysen werden separat für den Übergang zur ersten Ehe (Tabelle 3) sowie für die Geburt des ersten bzw. zweiten Kindes (Tabelle 4 und Tabelle 5) präsentiert. Die endgültigen Regressionsmodelle werden ausgehend von einem leeren Modell, das nur die Konstante und den regionalen Zufallseffekt enthält (Modell 1), schrittweise aufgebaut. Die individuellen Kontrollvariablen werden jeweils in Modell 2 bis 4 eingeführt; die Kontextvariablen folgen schließlich in den Modellen 5 bis 7. Die Schätzung der Modelle erfolgt unter Verwendung des Softwarepaketes $a M L$ (vgl. Lillard und Panis 2000).

\section{1 Übergang zur ersten Ehe}

Die Koeffizienten der individuellen Kontrollvariablen entsprechen den oben formulierten Erwartungen. Zusätzlich zu einem nicht-monotonen Alterseffekt (Modell 
2) zeigt sich, dass Frauen in Ausbildung eine geringere Neigung zur ersten Eheschließung aufweisen (Modell 3). Gleiches gilt für Frauen ohne Ausbildungsabschluss, wobei der entsprechende Koeffizient jedoch erst nach Kontrolle für das Vorhandensein eines Kindes statistisch signifikant wird (Modell 4). Dies deutet darauf hin, dass die niedrigere Heiratswahrscheinlichkeit von Frauen ohne Ausbildungsabschluss unterschätzt wird, wenn man nicht deren höheres Risiko einer vorehelichen Mutterschaft berücksichtigt, das $\mathrm{zu}$ einer höheren Heiratswahrscheinlichkeit unter Müttern ohne Ausbildungsabschluss führt. Die Einführung der Kind-Variable führt zu einer deutlichen Verbesserung der Güte des Modells und hat den erwarteten stark positiven Effekt auf die abhängige Variable. Neben ledigen Müttern weisen schließlich auch ausländische Frauen ein höheres Risiko zur Eheschließung auf (Modell 4), wenngleich der entsprechende Koeffizient nur auf dem 10-Prozent-Niveau signifikant ist.

Keine der in den Modellen 5 und 6 eingeführten Regionalvariablen stellt sich als statistisch signifikant heraus. Ein zwischenzeitlicher Anstieg des Land-Koeffizienten verschwindet nach Kontrolle für die Existenz einer dominierenden Wertorientierung (Modell 7). Der entsprechende Indikator ist schwach signifikant und hat überraschenderweise ein negatives Vorzeichen. Dies deutet auf unbeobachtete konfundierende sozio-kulturelle Einflüsse hin, die durch den regionalen Zufallseffekt $\left(\sigma_{u}\right)$ erfasst werden. Der ursprüngliche Wert von $\sigma_{u}$ vermindert sich nicht durch die Aufnahme individueller oder kontextueller Variablen in die Regression und ist in allen Modellen hochsignifikant, d.h. die Regressionskonstante variiert in Abhängigkeit vom jeweiligen Kontext.

\section{Tabelle 3}




\section{2 Übergang zum ersten und zweiten Kind}

Der Einfluss der Individualvariablen auf die Wahrscheinlichkeit ein Kind zu bekommen korrespondiert weitgehend mit den Befunden aus der Analyse des Eintritts in die erste Ehe. Hinsichtlich der Geburt des zweiten Kindes erweist sich darüber hinaus das Alter der Frau bei der ersten Geburt als wichtige erklärende Variable (Tabelle 5; Modell 2). Das positive Vorzeichen des Koeffizienten entspricht dabei den Erwartungen, da eine spätere erste Elternschaft weniger Spielraum für die Verschiebung weiterer Geburten lässt. ${ }^{4}$ Dieses Argument gilt auch für die Interpretation des Effektes der Bildungsvariablen auf das zweite Kind (Tabelle 5; Modell 3): Frauen mit Universitätsabschluss weisen eine höhere Wahrscheinlichkeit zu einer weiteren Geburt innerhalb des Beobachtungszeitraums auf, als Frauen mit einfachem Berufsabschluss. Blossfeld und Huinink (1991: 164f.) argumentieren bereits im Zusammenhang mit der ersten Geburt, dass eine längere Verweildauer im Bildungssystem dazu führt, dass Frauen mit hohen Ausbildungsabschlüssen unter Zeitdruck geraten, wenn sie die von ihnen angestrebte Kinderzahl realisieren wollen.

Schließlich ist das Risiko ein erstes oder zweites Kind $\mathrm{zu}$ bekommen bei verheirateten Frauen um ein Vielfaches höher, als bei unverheirateten Frauen. Die Berücksichtigung des Familienstands resultiert nicht nur in einer deutlich besseren Güte des jeweiligen Modells, sondern führt in der Analyse der ersten Geburt auch zu einer deutlichen Reduktion der anderen Koeffizienten (Tabelle 4; Modell 4). Dieser außerordentlich starke Effekt des Familienstandes korrespondiert eindeutig mit

\footnotetext{
${ }^{4}$ Die endgültige Kinderzahl einer Frau fällt mit höherem Erstgeburtsalter jedoch i.d.R. niedriger aus (z.B. Kohler et al. 2001).
} 
Ergebnissen anderer Untersuchungen, die auf eine weite Verbreitung kindorientierter Eheschließungen in Westdeutschland hinweisen (z.B. Matthias-Bleck 1997).

Hinsichtlich kontextueller Einflüsse auf das Risiko einer Mutterschaft lässt sich im sogenannten leeren Modell jeweils eine hochsignifikante regionale Zufallskomponente feststellen. Diese bleibt beim Übergang zum ersten Kind zwar nach Kontrolle für Alter und Bildung der Frau zunächst bestehen, verschwindet jedoch vollständig, sobald der Familienstand in die Regression aufgenommen wird (Tabelle 4; Modell 2 bis 4). Bei der Analyse der zweiten Geburt wird $\sigma_{u}$ bereits durch Inklusion der Altersvariablen insignifikant und auf ein Drittel seiner ursprünglichen Größe reduziert (Tabelle 5; Modell 2). Nichtsdestoweniger deutet anfangs der Koeffizient der Land-Variablen (jeweils in Modell 5) erwartungsgemäß darauf hin, dass Frauen in ländlichen Kreisen eine höhere Neigung zur Geburt eines ersten und zweiten Kindes haben. Dieser vielzitierte 'Effekt' kann jedoch durch Berücksichtigung weiterer Kontextvariablen weitgehend erklärt werden, d.h. er wird in den folgenden Modellen statistisch insignifikant, obwohl weder die regionalen Kinderbetreuungs- noch die Arbeitsmarktvariablen einen meßbaren eigenen Einfluß auf die Fertilitätsentscheidungen westdeutscher Frauen zeigen (jeweils in Modell 6). Bei der Analyse des Übergangs zum zweiten Kind verschwindet in den letzen beiden Modellen nicht nur die restliche latente regionale Heterogenität, sondern auch der Land-Koeffizient sinkt nach Aufnahme des Indikators für eine dominierende Wertorientierung auf nahezu Null (Tabelle 5; Modell 7). Letzteres deutet eher auf einen sozio-kulturellen als auf einen sozio-ökonomischen Ursprung der beobachteten Stadt-Land-Unterschiede hin.

Die Gegenüberstellung dieser Befunde mit den Resultaten einer Analyse auf der Ebene von Raumordnungsregionen (Hank 2002, im Druck) belegt die Gültigkeit der 
hier vorgestellten Ergebnisse, unabhängig von der räumlichen Definition des sozialen Kontexts.

\section{Tabelle 4}

\section{Tabelle 5}

\section{Zusammenfassung und Diskussion der Ergebnisse}

Die Berücksichtigung des (regionalen) sozialen Kontexts, in den die individuellen Akteure eingebettet sind, ist für jedes tragfähige Modell soziologischer Erklärung unentbehrlich (z.B. Coleman 1986; Esser 1988). In der vorliegenden Arbeit werden zunächst eine Reihe von Hypothesen über mögliche regionale Einflüsse auf die Familiengründung westdeutscher Frauen in den 1980er und 1990er Jahren formuliert, und anschließend empirisch getestet.

Die beiden wichtigsten Befunde der multivariaten Analyse sind erstens, dass unabhängig von der räumlichen Definition des sozialen Kontexts (vgl. Hank 2002, im Druck) regionale Unterschiede bezüglich der Geburt des ersten und des zweiten Kindes weitestgehend durch Kontrolle individueller Merkmale - vor allem Familienstand und Alter - erklärt werden können, und zweitens, dass es eine signifikante regionale Varianz im Heiratsverhalten gibt, die weder auf Kompositionseffekte noch auf strukturelle Kontexteffekte zurückzuführen ist.

Man kann davon ausgehen, dass regionsspezifische Unterschiede bei der Gründung von Partnerschaften und Ehen, die offenbar durch die Berücksichtigung der rohen Heiratsziffer im Modell nicht hinreichend erfasst werden, in umfassendere soziokulturelle Kontexte eingebettet sind. Eine Untersuchung des Zusammenhangs zwischen 
Heiratsalter und Kontrolle ehelicher Fruchtbarkeit in verschiedenen Weltregionen weist z.B. darauf hin, dass zumindest in traditionellen Gesellschaften soziale Kontexte in denen späte Eheschließungen die Norm sind, Frauen ein höheres Maß an individueller Autonomie und Kontrolle über ihr reproduktives Verhalten gewähren (vgl. Coale 1992). Selbst ohne Fortbestand der strukturellen Bedingungen, die ursprünglich die Verbreitung später Heiraten in einigen Regionen gefördert haben (z.B. durch bestimmte Erbschaftssysteme), erweisen sich räumliche Subkulturen demografischen Verhaltens häufig als sehr stabil über die Zeit (z.B. Lesthaeghe und Neels 2001; Reher 1998: 212ff.). Die räumliche Persistenz unterschiedlicher Muster der Familiengründung sollte dabei angesichts abnehmender direkter sozialer Regulierung zunehmend von regionalen Unterschieden in der Verbreitung allgemeiner Wertorientierungen (z.B. Lesthaeghe und Moors 2000) sowie ‘internalisierter Normen' bezüglich des Alters und der Reihenfolge, in der bestimmte Ereignisse stattfinden sollten, abhängen (vgl. Heckhausen 1999: 35). ${ }^{5}$

Für die westdeutsche Gesellschaft kann als grundsätzliche Erwartungshaltung unterstellt werden, dass der Ausbildungsabschluss vor der Eheschließung (z.B. Blossfeld und Huinink 1991) und die Eheschließung vor der Geburt des ersten Kindes (z.B. Billari und Kohler 2002, im Druck) erfolgen sollte. ${ }^{6}$ Obwohl längere Ausbildungszeiten allgemein zu einer Verzögerung der Familiengründung führen, kann in den hier geschätzten Modellen die regionale Varianz beim Eintritt in die erste Ehe und der Geburt des ersten Kindes aber nicht durch eine unterschiedliche Verteilung der

\footnotetext{
${ }^{5}$ Internalisierte Normen werden hier als relativ flexible Richtlinien für bestimmte familiäre Ereignisse verstanden, nicht als strikt einzuhaltende normative Grundsätze (vgl. Settersten und Hägestad 1996).

${ }^{6}$ Anders die Situation in Ostdeutschland, wo 1989 ein Drittel, und zehn Jahre später sogar die Hälfte aller Geburten außerhalb einer Ehe erfolgten (vgl. Konietzka und Kreyenfeld 2002, im Druck).
} 
individuellen Bildungsbeteiligung bzw. Bildungsabschlüsse erklärt werden. Ebensowenig erweisen sich Unterschiede hinsichtlich der individuellen Erfahrung einer vorehelichen Geburt als relevant für die Erklärung regional variierender Heiratsneigungen. Praktisch die gesamte regionale Heterogenität hinsichtlich der individuellen Wahrscheinlichkeit während des Beobachtungszeitraums ein erstes Kind zu bekommen wird jedoch durch Kontrolle des Familienstandes der Frau absorbiert. Dies deutet - zusammen mit dem Fehlen struktureller Kontexteffekte - auf die Existenz raumgebundener sozio-kultureller Milieus hin, die sich z.B. in der Akzeptanz nichtehelicher Lebensgemeinschaften und vorehelicher Elternschaft, oder in kollektiven Erwartungen hinsichtlich des Heiratsalters voneinander unterscheiden, was erst in der Konsequenz zu entsprechenden Unterschieden bei der Geburt des ersten und ggf. weiterer Kinder führt. Regionale Einflüsse auf das Geburtenverhalten haben somit keine eigenständige Qualität, sondern werden lediglich indirekt vermittelt, nämlich über einen direkten Kontexteffekt auf das Risiko einer ersten Eheschließung.

Trotz der Insignifikanz der Kinderbetreuungs- und Arbeitsmarktvariablen sollte aus den hier vorgelegten Befunden nicht geschlossen werden, dass regionale Opportunitätsstrukturen grundsätzlich keine Rolle für die individuelle Entscheidung zur Familiengründung spielen. Für das heutige Westdeutschland muss jedoch angenommen werden, dass - trotz spezifischer struktureller Profile von Regionen mit hohen und niedrigen Fertilitätsraten (vgl. Hank 2001) - in allen Kreisen eine so weitreichende Gleichheit der wirtschaftlichen und infrastrukturellen Lebensbedingungen besteht, dass Unterschiede darin die individuellen Kosten und den Nutzen von Kindern für ihre Eltern kaum beeinflussen. 
Leider konnten hier nur erste Hinweise auf die Art des Zusammenhangs zwischen regionalem Kontext und der Familiengründung westdeutscher Frauen gegeben werden. So kann z.B. nicht zwischen lokalen Gewohnheiten (dass in einem bestimmten Alter geheiratet wird) und lokalen Normen (dass in einem bestimmten Alter geheiratet werden sollte) unterschieden werden (z.B. Marini 1984). Eine genauere empirische Untersuchung verhaltensrelevanter Aspekte raumgebundener sozio-kultureller Milieus ist mit den üblichen Daten der sozialwissenschaftlichen Umfrageforschung jedoch nur sehr bedingt möglich (vgl. hierzu auch Hoffmeyer-Zlotnik [2000]). Ethnographische Studien einzelner Kontexte könnten daher zukünftig einen wichtigen Beitrag leisten, indem sie die notwendigen qualitativen Daten liefern, um regionsspezifische Verhaltenserwartungen, Wertorientierungen, etc. zu identifizieren und vergleichend zu analysieren. 


\section{Literatur}

Akademie für Raumforschung und Landesplanung (Hg.) 1983. Regionale Aspekte der Bevölkerungsentwicklung unter den Bedingungen des Geburtenrückgangs. Forschungs- und Sitzungsberichte, Bd. 144. Vincentz: Hannover.

Alexander, J.C. / B. Giesen / R. Münch / N.J. Smelser (Hrsg.). 1987. The Micro-Macro Link. Berkley: University of California Press.

Allison, P.A. 1982. Discrete-Time Methods for the Analysis of Event Histories. In: S. Leinhardt (Hg.), Sociological Methodology 12. San Francisco: Jossey-Bass, 6198.

Bähr, J. / P. Gans (Hrsg.). 1991. The Geographical Approach to Fertility. Kieler Geographische Schriften, Bd. 78. Kiel.

Barber, J.S. / S.A. Murphy / W.G. Axinn / J. Marples. 2000. Discrete-time multilevel hazard analysis. In: M.E. Sobel / M.P. Becker (Hrsg.), Sociological Methodology 30. Washington: Blackwell Publishers, 201-235.

Becker, G.S. 1993. A Treatise on the Family (Erweiterte Auflage). Cambridge: Harvard University Press.

Bertram, H. 1996. Familienentwicklung und Haushaltsstrukturen. In: Strubelt W, et al. (Hrsg.), Städte und Regionen - Räumliche Folgen des Transformationsprozesses. Leske + Budrich: Opladen, 183-215.

Bertram, H. / Nauck, B. / Klein, T. (Hrsg.). 2000. Solidarität, Lebensformen und regionale Entwicklung. Leske + Budrich: Opladen.

Billari, F.C. / H.-P. Kohler. 2002. The impact of union formation dynamics on first births in West Germany and Italy: Are there signs of convergence? In: E. Klijzing / M. Corijn (Hrsg.), Comparative Research on Fertility and the Family in Contemporary Europe: Findings and Lessons. New York and Geneva: United Nations, im Druck.

Billy, J.O. / D.E. Moore. 1992. A multilevel analysis of marital and nonmarital fertility in the US. Social Forces 70: 977-1011. 
Blossfeld, H.-P. 1987. Labor-Market Entry and the Sexual Segregation of Careers in the Federal Republic of Germany. American Journal of Sociology 93: 89-118.

Blossfeld, H.-P. / J. Huinink. 1991. Human Capital Investments or Norms of Role Transition? How Women's Schooling and Career Affect the Process of Family Formation. American Journal of Sociology 97: 143-168.

Brewster, K.L. / R.R. Rindfuss. 2000. Fertility and Women's Employment in Industrialized Nations. Annual Review of Sociology 26: 271-296.

Coale, A.J. 1992. Age of Entry into Marriage and the Date of the Initiation of Voluntary Birth Control. Demography 29: 333-341.

Coleman, J.S. 1986. Social Theory, Social Research, and a Theory of Action. American Journal of Sociology 91: 1309-13035.

Coleman, J.S. 1990. Foundations of Social Theory. Cambridge: Harvard University Press.

Conrad C. / Lechner, M. / Werner, W. 1996. East German fertility after unification: crisis or adaptation? Population and Development Review 22: 331-358.

Courgeau, D. / Baccaini, B. 1998. Multilevel analysis in the social sciences. Population (English Selection) 10: 39-71.

DiPrete, T.A. / J.D. Forristal. 1994. Multilevel Models - Methods and Substance. Annual Review of Sociology 20: 331-357.

Eberstadt, N. 1994: Demographic Shocks after Communism: Eastern Germany, 198993. Population and Development Review 20: 137-151.

Erbring, L. / A.A. Young. 1979. Individuals and Social Structure. Contextual Effects as Endogenous Feedback. Sociological Methods \& Research 7: 396-430.

Esser, H. 1988. Sozialökologische Stadtforschung und Mehr-Ebenen-Analyse. In: J. Friedrichs (Hg.), Soziologische Stadtforschung, Kölner Zeitschrift für Soziologie und Sozialpsychologie, Sonderheft 29: 33-55.

Esser, H. 1996. Soziologie. Allgemeine Grundlagen (2. Auflage). Frankfurt und New York: Campus. 
Esser, H. 1998. Why are Bridge Hypotheses Necessary? In: H.-P. Blossfeld / G. Prein (Hrsg.), Rational Choice Theory and Large-Scale Data Analysis. Boulder: Westview Press, 94-111.

Gans, P. 1996. Demographische Entwicklung seit 1980. In: W. Strubelt et al. (Hrsg.) Städte und Regionen - Räumliche Folgen des Transformationsprozesses. Leske + Budrich: Opladen, 141-181.

Guo, G. / H. Zhao. 2000. Multilevel Modeling for Binary Data. Annual Review of Sociology 26: 441-462.

Hank, K. 2001. Regional Fertility Differences in Western Germany: An Overview of the Literature and Recent Descriptive Findings. International Journal of Population Geography 7: 243-257.

Hank, K. 2002. Regional Social Contexts and Individual Fertility Decisions: A Multilevel Analysis of First and Second Births in Western Germany. European Journal of Population 18, im Druck.

Hank, K. / M. Kreyenfeld. 2002. Modes of Childcare and the Difficult Compatibility of Childrearing and Emplyoment in (Western) Germany. In: Materialien zur Bevölkerungswissenschaft, Heft 105, im Druck.

Hauser, R.M. 1974. Contextual Analysis Revisited. Sociological Methods \& Research 2 : 365-375.

Hechter, M. / S. Kanazawa. 1997. Sociological Rational Choice Theory. Annual Review of Sociology 23: 191-214.

Heckhausen, J. 1999. Developmental Regulation in Adulthood. Age-Normative and Sociostructural Constraints as Adaptive Challenges. Cambridge: Cambridge University Press.

Hernes, G. 1976. Structural Change in Social Processes. American Journal of Sociology 82: 513-547.

Hoem, B. 2000. Entry into motherhood in Sweden: the influence of economic factors on the rise and fall in fertility, 1986-1997. Demographic Research [Online] 2, 
verfügbar http://www.demographic-research.org/Volumes/Vol2/4.

Hoffmeyer-Zlotnik, J.H.P. 2000. Regionalisierung sozialwissenschaftlicher Umfragedaten. Opladen: Westdeutscher Verlag.

Huber, J. (Hg.). 1991. Macro-Micro Linkages in Sociology. Newbury Park: Sage.

Huinink, J. / M. Wagner. 1989. Regionale Lebensbedingungen, Migration und Familienbildung. Kölner Zeitschrift für Soziologie und Sozialpsychologie 41: 669-689.

Knodel, J.E. 1974. The Decline of Fertility in Germany, 1871-1939. Princeton University Press: Princeton.

Knodel, J.E. 1988. Demographic behavior in the past: A study of fourteen German village populations in the eighteenth and nineteenth centuries. Cambridge University Press: Cambridge.

Kohler, H.-P. / A. Skytthe / K. Christensen (2001): The Age at First Birth and Completed Fertility Reconsidered: Findings from a Sample of Identical Twins. MPIDR Working Paper WP-2001-006, verfügbar Http://www.demogr.mpg.de/Papers/Working/wp-2001-006.pdf.

Konietzka, D. / M. Kreyenfeld. 2002. Women's Employment and Non-Marital Childbearing: A Comparison of East and West Germany in the 1990s. Population 57, im Druck.

Kopp, J. 2000. Geburtenentwicklung in Ost- und Westdeutschland. In: H. Bertram et al. (Hrsg.), Solidarität, Lebensformen und regionale Entwicklung. Opladen: Leske+Budrich, 83-135.

Kravdal, Ø. 1996. How the local supply of day-care centers influences fertility in Norway: A parity-specific approach. Population Research and Policy Review 15: 201-218.

Kravdal, Ø. 2002. The impact of individual and aggregate unemployment on fertility in Norway. Demographic Research [Online] 6, verfügbar http://www.demographicresearch.org/Volumes/Vol6/10.

Lazarsfeld, P.F. / H. Menzel. 1969. On the relations between individual and collective 
properties. In: A. Etzioni (Hg.), A sociological reader on complex organizations. New York: Holt, Rinehart \& Winston, 499-516.

Lestaheghe, R. / G. Moors. 2000. Life course transitions and value orientations: selection and adaptation. Interuniversity Papers in Demography WP 2000-7, verfügbar http://www.vub.ac.be/SOCO/IPDWP2000-7.pdf.

Lesthaeghe, R. / K. Neels. 2001. From the First to the Second Demographic Transition: an Interpretation of the Spatial Continuity of Demographic Innovation in France, Belgium and Switzerland. Vortrag bei der Euresco-Konferenz 'The Second Demographic transition in Europe', Bad Herrenalb, 23.-28. Juni.

Lesthaeghe, R. / J. Surkyn. 1988. Cultural Dynamics and Economic Theories of Fertility Change. Population and Development Review 14: 1-45.

Lichter, D.T. / F.B. LeClere / D.K. McLaughlin. 1991. Local Marriage Markets and the Marital Behavior of Black and White Women. American Journal of Sociology 96: 843-867.

Lillard, L.A. / C.W.A. Panis. 2000. aML Multilevel Multiprocess Statistical Software. Release 1.0. Los Angeles: EconWare.

Lindenberg, S. 1977. Individuelle Effekte, kollektive Phänomene und das Problem der Transformation. In: K. Eichner / W. Habermehl (Hrsg.), Probleme der Erklärung sozialen Verhaltens. Meisenheim: Hain, 46-84.

Lindenberg, S. 1990. Homo Socio-Oeconomicus: The Emergence of a General Model of Man in the Social Sciences. Journal of Institutional and Theoretical Economics 146: $727-748$.

Manski, C.F. 1993. Identification of Endogenous Social Effects: The Reflection Problem. The Review of Economic Studies 60: 531-542.

Marini, M.M. 1984. Age and Sequencing Norms in the Transition to Adulthood. Social Forces 63: 229-244.

Matthias-Bleck, H. 1997. Warum noch Ehe? Erklärungsversuche der kindorientierten Eheschließung. Bielefeld. 
Münch, R. / N.J. Smelser. 1987. Relating the Micro and Macro. In: J.C. Alexander et al. (Hrsg.), The Micro-Macro Link. Berkley: University of California Press, 356-388.

Nauck B. 1995: Regionale Millieus von Familien in Deutschland nach der politischen Vereinigung. In: B. Nauck / C. Onnen-Isemann (Hrsg.), Familie im Brennpunkt. Luchterhand: Neuwied, 91-121.

Reher, D.S. 1998. Family Ties in Western Europe: Persistent Contrasts. Population and Development Review 24: 203-234.

Settersten, R.A. / G.O. Hägestad. 1996. What's the Latest? Cultural Age Deadlines for Family Transitions. The Gerontologist 36: 178-188.

Snijders, T.A.B. / R.J. Bosker. 1999. Multilevel analysis: An introduction to basic and advanced multilevel modeling. London: Sage.

SOEP-Gruppe. 2001. The German Socio-Economic Panel (GSOEP) after more than 15 years - Overview. In: E. Holst et al. (Hrsg.), Proceedings of the 2000 Fourth International Conference of German Socio-Economic Panel Study Users (GSOEP2000), Vierteljahrshefte zur Wirtschaftsforschung 70: 7-14.

South, S.J. / K.D. Crowder (1999): Neighborhood effects on family formation: concentrated poverty and beyond. American Sociological Review 64: 113-132.

South, S.J. / K.M. Lloyd. 1992. Marriage Markets and Nonmarital Fertility in the United States. Demography 29: 247-264.

Teachman, J. / K. Crowder. 2002. Multilevel Models in Family Research: Some Conceptual and Methodological Issues. Journal of Marriage and the Family 64: 280-294.

Yamaguchi, K. 1991. Event History Analysis. Newbury Park: Sage. 


\section{Anhang}

Abbildung 1: Ein Mehrebenenmodell individuellen Handelns

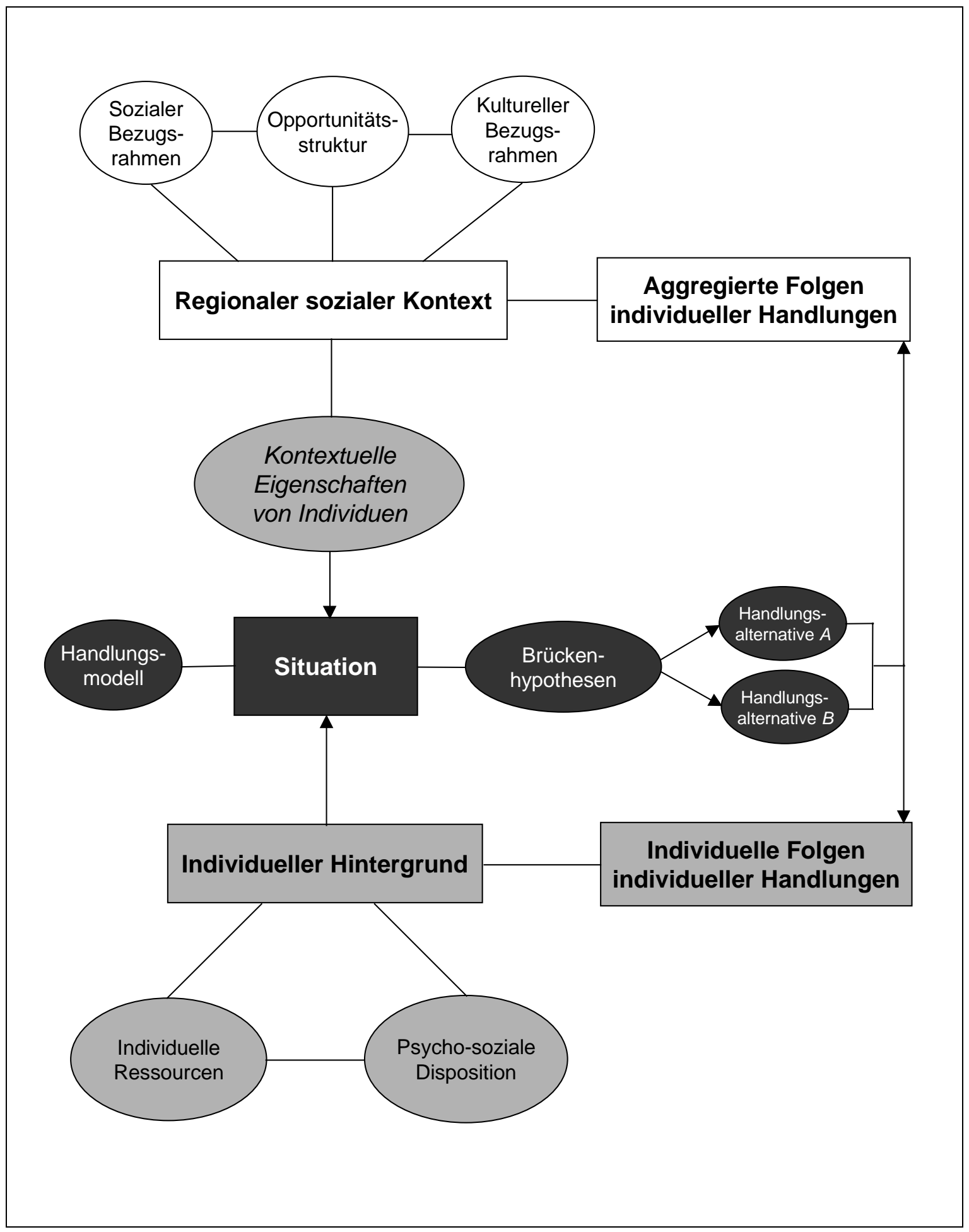

Quelle: eigene Darstellung 
Tabelle 1: Variablenbeschreibung

\begin{tabular}{|c|c|}
\hline \multicolumn{2}{|r|}{ Abhängige Variablen } \\
\hline Heirat / Geburt & $\begin{array}{l}\text { Binäre Variablen die den Wert } 1 \text { annehmen, wenn die Frau innerhalb } \\
\text { eines Ein-Jahres-Intervalls der Periode 1984-1999 heiratet bzw. ein } \\
\text { erstes oder zweites Kind bekommt. }\end{array}$ \\
\hline \multicolumn{2}{|r|}{ Individualmerkmale } \\
\hline Alter & Alter, quadriertes Alter, und Alter bei Geburt des ersten Kindes. \\
\hline Bildung & $\begin{array}{l}\text { Zeitabhängige binäre Variablen, die den höchsten Bildungsabschluss } \\
\text { zum Zeitpunkt des jeweiligen Interviews angeben: in Ausbildung, } \\
\text { kein Ausbildungsabschluss, beruflicher Ausbildungsabschluss } \\
\text { (Referenzkategorie), Universitätsabschluss. }\end{array}$ \\
\hline Kind $^{\mathrm{a}}$ & $\begin{array}{l}\text { Zeitabhängige binäre Variable die den Wert } 1 \text { annimmt, wenn die } \\
\text { Frau Mutter (mindestens) eines Kindes ist. }\end{array}$ \\
\hline Familienstand $^{\mathrm{b}}$ & $\begin{array}{l}\text { Zeitabhängige binäre Variable die den Wert } 1 \text { annimmt, wenn die } \\
\text { Frau verheiratet ist. }\end{array}$ \\
\hline Ausländerin & $\begin{array}{l}\text { Zeitunabhängige Variable die den Wert } 1 \text { annimmt, wenn die Frau } \\
\text { zur AusländerInnen-Stichprobe des SOEP gehört. }\end{array}$ \\
\hline \multicolumn{2}{|r|}{ Regionalmerkmale } \\
\hline Urbanisierungsgrad & $\begin{array}{l}\text { Zeitunabhängige binäre Variablen, die angeben, ob ein Kreis als } \\
\text { Agglomerationsraum (Referenzkategorie), verstädterter Raum, oder } \\
\text { ländlicher Raum definiert ist. }\end{array}$ \\
\hline Kinderbetreuung $^{\mathrm{b}}$ & $\begin{array}{l}\text { Regionale Versorgung mit Betreuungsplätzen in Kindergärten pro } \\
1000 \text { Kinder im Alter von 3-6 (zeitabhängig, 1986/1994). }\end{array}$ \\
\hline Männer-Anteil $^{\mathrm{a}}$ & $\begin{array}{l}\text { Durchschnittlicher Männer-Anteil an der Bevölkerung im Alter von } \\
25 \text { bis } 30 \text { Jahren in der Periode 1995-1997 (in \%) (zeitunabhängig). }\end{array}$ \\
\hline Dienstleistungssektor & $\begin{array}{l}\text { Anteil der Beschäftigten im Handel (in \%) (zeitabhängig, } \\
\text { 1987/1995). }\end{array}$ \\
\hline Arbeitslosenquote & Regionale Arbeitslosenquote (in \%) (zeitabhängig, 1987/1996). \\
\hline Frauenerwerbsquote & Regionale Frauenerwerbsquote (in \%) (zeitabhängig, 1987/1995). \\
\hline Rohe Heiratsziffer $^{a}$ & $\begin{array}{l}\text { Durchschnittliche rohe Heiratsziffer der Jahre } 1986 \text { und } 1993 \\
\text { (zeitunabhängig). }\end{array}$ \\
\hline Rohe Geburtenziffer $^{b}$ & $\begin{array}{l}\text { Durchschnittliche rohe Geburtenziffer der Jahre } 1989 \text { und } 1995 \\
\text { (zeitunabhängig). }\end{array}$ \\
\hline $\begin{array}{l}\text { Indikator für dominierende } \\
\text { Wertorientierung }\end{array}$ & $\begin{array}{l}\text { Zeitunabhängige binäre Variable die den Wert } 1 \text { annimmt, wenn eine } \\
\text { Partei mehr als } 50 \text { Prozent der Stimmen bei den beiden letzten } \\
\text { Wahlen (Europaparlament 1989, Landtag 1995/1999) erhalten hat } \\
\text { und mehr als zwei Drittel der Bevölkerung der selben Konfession } \\
\text { angehören. }\end{array}$ \\
\hline \multicolumn{2}{|c|}{$\begin{array}{l}\text { Anmerkungen: } \\
{ }^{\mathrm{a}} \text { Variable wird nur in der Heiratsanalyse verwendet. } \\
{ }^{\mathrm{b}} \text { Variable wird nur in der Fertilitätsanalyse verwendet. }\end{array}$} \\
\hline
\end{tabular}


Tabelle 2: Deskriptive Statistiken

\begin{tabular}{|c|c|c|c|}
\hline Variablen & 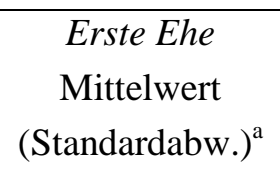 & 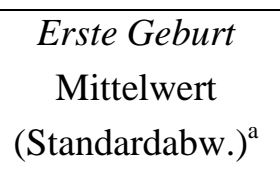 & $\begin{array}{c}\text { Zweite Geburt } \\
\text { Mittelwert } \\
\text { (Standardabw.) }^{\mathrm{a}}\end{array}$ \\
\hline Individualmerkmale & & & \\
\hline Alter & $24.8(3.9)$ & $25.5(4.1)$ & $31.9(4.4)$ \\
\hline Quadriertes Alter & $629.9(206.8)$ & $665.0(218.1)$ & $1036.0(283.7)$ \\
\hline Alter bei erster Geburt & - & - & $25.2(4.1)$ \\
\hline In Ausbildung & .22 & .17 & .01 \\
\hline Kein Ausbildungsabschluss & .17 & .18 & .24 \\
\hline Berufl. Ausbildungsabschluss & .53 & .57 & .67 \\
\hline Universitätsabschluss & .07 & .08 & .06 \\
\hline Kind & .09 & - & - \\
\hline Familienstand & - & .27 & .80 \\
\hline Ausländerin & .22 & .23 & .24 \\
\hline Regionalmerkmale & & & \\
\hline Agglomerationsraum & .59 & .60 & .57 \\
\hline Verstädterter Raum & .25 & .25 & .26 \\
\hline Ländlicher Raum & .15 & .15 & .17 \\
\hline Männer-Anteil (25-30 Jahre) & $51.3(1.2)$ & - & - \\
\hline Kinderbetreuung & - & $825.5(175.0)$ & $830.1(174.3)$ \\
\hline Dienstleistungssektor & $17.2(3.9)$ & $17.2(3.9)$ & $17.2(4.0)$ \\
\hline Arbeitslosenquote & $8.8(3.2)$ & $8.7(3.2)$ & $8.6(3.0)$ \\
\hline Frauenerwerbsquote & $41.4(3.5)$ & $41.2(3.5)$ & $41.2(3.5)$ \\
\hline Rohe Heiratsziffer & $7.5(0.5)$ & - & - \\
\hline Rohe Geburtenziffer & - & $10.2(.9)$ & $10.2(.9)$ \\
\hline Indikator für Wertorientierung & .10 & .10 & .09 \\
\hline $\mathrm{N}$ (Ereignisse) & 746 & 1025 & 681 \\
\hline N (Kreise) & 288 & 300 & 273 \\
\hline $\mathrm{N}$ (Frauen) & 2266 & 2892 & 1585 \\
\hline $\mathrm{N}$ (Beobachtungen) & 10077 & 13537 & 6389 \\
\hline
\end{tabular}

Quelle: SOEP 1984-1999, DJI Regionaldatenbank, Statistik regional 1999, eigene Berechnungen 
Tabelle 3: 'Erste Ehe' - Ergebnisse der zeitdiskreten logistischen Mehrebenenmodelle, 1984-1999

\begin{tabular}{|c|c|c|c|c|c|c|c|c|c|c|c|c|c|c|c|c|c|c|c|c|c|}
\hline & \multicolumn{3}{|c|}{ Modell 1} & \multicolumn{3}{|c|}{ Modell 2} & \multicolumn{3}{|c|}{ Modell 3} & \multicolumn{3}{|c|}{ Modell 4} & \multicolumn{3}{|c|}{ Modell 5} & \multicolumn{3}{|c|}{ Modell 6} & \multicolumn{3}{|c|}{ Modell 7} \\
\hline & $\beta$ & s.e. & Sig. & $\beta$ & s.e. & Sig. & $\beta$ & s.e. & Sig. & $\beta$ & s.e. & Sig. & $\beta$ & s.e. & Sig. & $\beta$ & s.e. & Sig. & $\beta$ & s.e. & Sig. \\
\hline Alter & - & & & 1.19 & .16 & $* * *$ & .98 & .16 & $* * *$ & 1.00 & .17 & $* * *$ & 1.00 & .17 & $* * *$ & 1.03 & .17 & $* * *$ & 1.03 & .17 & $* * *$ \\
\hline Quadriertes Alter & - & & & -.02 & .00 & $* * *$ & -.02 & .00 & $* * *$ & -.02 & .00 & $* * *$ & -.02 & .00 & $* * *$ & -.02 & .00 & $* * *$ & -.02 & .00 & $* * *$ \\
\hline In Ausbildung ${ }^{\mathrm{a}}$ & - & & & - & & & -1.18 & .16 & $* * *$ & -1.18 & .16 & $* * *$ & -1.15 & .16 & $* * *$ & -1.14 & .16 & $* * *$ & -1.14 & .16 & $* * *$ \\
\hline Kein Abschluss $^{\mathrm{a}}$ & - & & & - & & & -.11 & .11 & & -.31 & .11 & $* * *$ & -.31 & .11 & $* * *$ & -.32 & .11 & $* * *$ & -.32 & .11 & $* * *$ \\
\hline Univ.-abschluss $^{\mathrm{a}}$ & - & & & - & & & -.06 & .15 & & -.06 & .15 & & .06 & .15 & & .06 & .16 & & .06 & .16 & \\
\hline Kind & - & & & - & & & - & & & 1.00 & .09 & $* * *$ & 1.01 & .10 & $* * *$ & 1.02 & .10 & $* * *$ & 1.02 & .10 & $* * *$ \\
\hline Ausländerin & - & & & - & & & - & & & .19 & .10 & $*$ & .18 & .10 & * & .20 & .11 & * & .19 & .11 & $*$ \\
\hline Verstädterter Raum ${ }^{\mathrm{b}}$ & - & & & - & & & - & & & - & & & -.05 & .11 & & -.11 & .12 & & -.08 & .13 & \\
\hline Ländlicher Raum ${ }^{\mathrm{b}}$ & - & & & - & & & - & & & - & & & -.02 & .13 & & -.12 & .14 & & .02 & .19 & \\
\hline Männer-Anteil & - & & & - & & & - & & & - & & & - & & & .01 & .04 & & .02 & .04 & \\
\hline Dienstleistungsektor & - & & & - & & & - & & & - & & & - & & & -.01 & .02 & & -.01 & .02 & \\
\hline Arbeitslosenquote & - & & & - & & & - & & & - & & & - & & & -.02 & .02 & & -.02 & .02 & \\
\hline Frauenerwerbsquote & - & & & - & & & - & & & - & & & - & & & -.01 & .01 & & -.02 & .02 & \\
\hline Rohe Heiratsziffer & - & & & - & & & - & & & - & & & - & & & - & & & .01 & .06 & \\
\hline Wertorientierung & - & & & - & & & - & & & - & & & - & & & - & & & -.34 & .21 & $*$ \\
\hline Konstante & -2.52 & .04 & $* * *$ & -18.37 & 2.05 & $* * *$ & -15.03 & 2.11 & $* * *$ & -15.28 & 2.19 & $* * *$ & -15.25 & 2.19 & $* * *$ & -14.44 & 2.24 & $* * *$ & -14.36 & 2.42 & $* * *$ \\
\hline$\sigma_{u}$ & .24 & .09 & $* * *$ & .29 & .09 & $* * *$ & .28 & .09 & $* * *$ & .33 & .09 & $* * *$ & .33 & .09 & $* * *$ & .34 & .09 & $* * *$ & .32 & .09 & $* * *$ \\
\hline-2 Log likelihood ${ }^{\mathrm{c}}$ & 5316 & & - & 5221 & & $* * *$ & 5136 & & $* * *$ & 5058 & & $* * *$ & 5058 & & & 5051 & & & 5047 & & \\
\hline \multicolumn{22}{|c|}{$\begin{array}{l}\text { Anmerkungen: } \\
{ }^{\mathrm{a}} \text { Referenzkategorie: beruflicher Ausbildungsabschluss. } \\
{ }^{\mathrm{b}} \text { Referenzkategorie: Agglomerationsraum. } \\
{ }^{\mathrm{c}} \text { Signifikanztest für }-2 \text { Log likelihood im Vergleich zum vorherigen Modell. } \\
\text { Signifikanz: } *<.10 ; * *<.05 ; * * *<.01\end{array}$} \\
\hline
\end{tabular}

Quelle: SOEP 1984-1999, DJI Regionaldatenbank, Statistik regional 1999, eigene Berechnungen 
Tabelle 4: 'Erste Geburt' - Ergebnisse der zeitdiskreten logistischen Mehrebenenmodelle, 1984-1999

\begin{tabular}{|c|c|c|c|c|c|c|c|c|c|c|c|c|c|c|c|c|c|c|c|c|c|}
\hline & \multicolumn{3}{|c|}{ Modell 1} & \multicolumn{3}{|c|}{ Modell 2} & \multicolumn{3}{|c|}{ Modell 3} & \multicolumn{3}{|c|}{ Modell 4} & \multicolumn{3}{|c|}{ Modell 5} & \multicolumn{3}{|c|}{ Modell 6} & \multicolumn{3}{|c|}{ Modell 7} \\
\hline & $\beta$ & s.e. & Sig. & $\beta$ & s.e. & Sig. & $\beta$ & s.e. & Sig. & $\beta$ & s.e. & Sig. & $\beta$ & s.e. & Sig. & $\beta$ & s.e. & Sig. & $\beta$ & s.e. & Sig. \\
\hline Alter & - & & & .92 & .11 & $* * *$ & .75 & .12 & $* * *$ & .37 & .12 & $* * *$ & .38 & .13 & $* * *$ & .39 & .13 & $* * *$ & .39 & .13 & $* * *$ \\
\hline Quadriertes Alter & - & & & -.02 & .00 & $* * *$ & -.01 & .00 & $* * *$ & -.01 & .00 & $* * *$ & -.01 & .00 & $* * *$ & -.01 & .00 & $* * *$ & -.01 & .00 & $* * *$ \\
\hline In Ausbildung ${ }^{\mathrm{a}}$ & - & & & - & & & -1.53 & .19 & $* * *$ & -.86 & .20 & $* * *$ & -.84 & .19 & $* * *$ & -.84 & .20 & $* * *$ & -.84 & .20 & $* * *$ \\
\hline Kein Abschluss ${ }^{\mathrm{a}}$ & - & & & - & & & .33 & .09 & $* * *$ & .13 & .09 & & .13 & .09 & & .13 & .09 & & .13 & .09 & \\
\hline Univ.-abschluss $^{\mathrm{a}}$ & - & & & - & & & -.15 & .13 & & .14 & .14 & & .17 & .14 & & .18 & .14 & & .18 & .14 & \\
\hline Familienstand & - & & & - & & & - & & & 2.55 & .08 & $* * *$ & 2.55 & .08 & $* * *$ & 2.55 & .08 & $* * *$ & 2.55 & .08 & $* * *$ \\
\hline Ausländerin & - & & & - & & & - & & & .06 & .09 & & .09 & .09 & & .11 & .09 & & .11 & .09 & \\
\hline Verstädterter Raum ${ }^{\mathrm{b}}$ & - & & & - & & & - & & & - & & & .09 & .09 & & .10 & .09 & & .09 & .10 & \\
\hline Ländlicher Raum ${ }^{\mathrm{b}}$ & - & & & - & & & - & & & - & & & .21 & .10 & $* *$ & .20 & .11 & $*$ & .17 & .11 & \\
\hline Kinderbetreuung & - & & & - & & & - & & & - & & & - & & & -.00 & .00 & & -.00 & .00 & \\
\hline Dienstleistungsektor & - & & & - & & & - & & & - & & & - & & & -.01 & .01 & & -.01 & .01 & \\
\hline Arbeitslosenquote & - & & & - & & & - & & & - & & & - & & & -.00 & .01 & & -.00 & .01 & \\
\hline Frauenerwerbsquote & - & & & - & & & - & & & - & & & - & & & -.01 & .01 & & -.01 & .01 & \\
\hline Rohe Geburtenziffer & - & & & - & & & - & & & - & & & - & & & - & & & .01 & .05 & \\
\hline Wertorientierung & - & & & - & & & - & & & - & & & - & & & - & & & .03 & .17 & \\
\hline Konstante & -2.50 & .04 & $* * *$ & -15.34 & 1.52 & $* * *$ & -12.68 & 1.56 & $* * *$ & -8.10 & 1.62 & $* * *$ & -8.28 & 1.69 & $* * *$ & -7.58 & 1.70 & $* * *$ & -7.71 & 1.77 & $* * *$ \\
\hline$\sigma_{u}$ & .25 & .06 & $* * *$ & .29 & .06 & $* * *$ & .25 & .06 & $* * *$ & .00 & - & & .00 & - & & .00 & - & & .00 & - & \\
\hline-2 Log likelihood $^{\mathrm{c}}$ & 7251 & & - & 7135 & & $* * *$ & 6983 & & $* * *$ & 5822 & & $* * *$ & 5817 & & $*$ & 5813 & & & 5813 & & \\
\hline \multicolumn{22}{|c|}{$\begin{array}{l}\text { Anmerkungen: } \\
\text { a Referenzkategorie: beruflicher Ausbildungsabschluss. } \\
{ }^{\mathrm{b}} \text { Referenzkategorie: Agglomerationsraum. } \\
{ }^{\mathrm{c}} \text { Signifikanztest für }-2 \text { Log likelihood im Vergleich zum vorherigen Modell. } \\
\text { Signifikanz: } *<.10 ; * *<.05 ; * * *<.01\end{array}$} \\
\hline
\end{tabular}

Quelle: SOEP 1984-1999, DJI Regionaldatenbank, Statistik regional 1999, eigene Berechnungen 
Tabelle 5: 'Zweite Geburt' - Ergebnisse der zeitdiskreten logistischen Mehrebenenmodelle, 1984-1999

\begin{tabular}{|c|c|c|c|c|c|c|c|c|c|c|c|c|c|c|c|c|c|c|c|c|c|}
\hline & \multicolumn{3}{|c|}{ Modell 1} & \multicolumn{3}{|c|}{ Modell 2} & \multicolumn{3}{|c|}{ Modell 3} & \multicolumn{3}{|c|}{ Modell 4} & \multicolumn{3}{|c|}{ Modell 5} & \multicolumn{3}{|c|}{ Modell 6} & \multicolumn{3}{|c|}{ Modell 7} \\
\hline & $\beta$ & s.e. & Sig. & $\beta$ & s.e. & Sig. & $\beta$ & s.e. & Sig. & $\beta$ & s.e. & Sig. & $\beta$ & s.e. & Sig. & $\beta$ & s.e. & Sig. & $\beta$ & s.e. & Sig. \\
\hline & - & & & .59 & .18 & $* * *$ & .55 & .19 & $* * *$ & .59 & .19 & $* * *$ & .60 & .19 & $* * *$ & .61 & .19 & $* * *$ & .61 & .19 & $* * *$ \\
\hline Alter & & & & & & & & & & & & & & & & & & & & & \\
\hline Quadriertes Alter & - & & & -.01 & .00 & $* * *$ & -.01 & .00 & $* * *$ & -.01 & .00 & $* * *$ & -.01 & .00 & $* * *$ & -.01 & .00 & $* * *$ & -.01 & .00 & $* * *$ \\
\hline Alter bei 1 . Geburt & - & & & .12 & .01 & $* * *$ & .11 & .01 & $* * *$ & .09 & .01 & $* * *$ & .09 & .01 & $* * *$ & .09 & .01 & $* * *$ & .09 & .01 & $* * *$ \\
\hline In Ausbildung ${ }^{\mathrm{a}}$ & - & & & - & & & -.88 & .60 & & -.55 & .65 & & -.53 & .65 & & -.50 & .65 & & -.50 & .66 & \\
\hline Kein Abschluss ${ }^{a}$ & - & & & - & & & -.05 & .09 & & -.07 & .10 & & -.07 & .10 & & -.07 & .10 & & -.07 & .10 & \\
\hline Univ.-abschluss $^{\mathrm{a}}$ & - & & & - & & & .48 & .16 & $* * *$ & .51 & .16 & $* * *$ & .54 & .16 & $* * *$ & .54 & .16 & $* * *$ & .55 & .16 & $* * *$ \\
\hline Familienstand & - & & & - & & & - & & & 1.49 & .18 & $* * *$ & 1.50 & .19 & $* * *$ & 1.48 & .19 & $* * *$ & 1.49 & .19 & $* * *$ \\
\hline Ausländerin & - & & & - & & & - & & & .09 & .11 & & .13 & .12 & & .12 & .12 & & .13 & .12 & \\
\hline Verstädterter Raum ${ }^{\mathrm{b}}$ & - & & & - & & & - & & & - & & & -.02 & .10 & & -.06 & .11 & & -.13 & .12 & \\
\hline Ländlicher Raum ${ }^{\text {b }}$ & - & & & - & & & - & & & - & & & .22 & .11 & $*$ & .18 & .13 & & .01 & .15 & \\
\hline Kinderbetreuung & - & & & - & & & - & & & - & & & - & & & .00 & .00 & & .00 & .00 & \\
\hline Dienstleistungsektor & - & & & - & & & - & & & - & & & - & & & -.00 & .02 & & -.00 & .02 & \\
\hline Arbeitslosenquote & - & & & - & & & - & & & - & & & - & & & -.02 & .02 & & -.02 & .02 & \\
\hline Frauenerwerbsquote & - & & & - & & & - & & & - & & & - & & & -.00 & .01 & & -.00 & .02 & \\
\hline Rohe Geburtenziffer & - & & & - & & & - & & & - & & & - & & & - & & & .07 & .06 & \\
\hline Wertorientierung & - & & & - & & & - & & & - & & & - & & & - & & & .21 & .18 & \\
\hline Konstante & -2.14 & .05 & $* * *$ & -11.48 & 2.79 & $* * *$ & -10.67 & 2.89 & $* * *$ & -12.35 & 2.92 & $* * *$ & -12.54 & 2.92 & $* * *$ & -12.31 & 2.93 & $* * *$ & -13.21 & 3.00 & $* * *$ \\
\hline$\sigma_{u}$ & .42 & .08 & $* * *$ & .12 & .16 & & .08 & .23 & & .08 & .25 & & .06 & .33 & & .00 & - & & .00 & - & \\
\hline-2 Log likelihood $^{\mathrm{c}}$ & 4320 & & 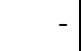 & 4058 & & $* * *$ & 4045 & & $* *$ & 3950 & & $* * *$ & 3946 & & & 3942 & & & 3938 & & \\
\hline $\begin{array}{l}\text { Anmerkungen: } \\
\text { a Referenzkategorie: } \\
{ }^{\mathrm{b}} \text { Referenzkategorie: } \\
{ }^{\mathrm{c}} \text { Signifikanztest für - } \\
\text { Signifikanz: } *<.10 \text {; }\end{array}$ & $\begin{array}{l}\text { ruflich } \\
\text { gglom } \\
\text { Log li } \\
* *<.05\end{array}$ & $\begin{array}{l}\text { lihoo } \\
* * *<\end{array}$ & $\mathrm{m}$. & $\begin{array}{l}\text { abschl } \\
\text { rgleich }\end{array}$ & $\mathrm{m} v$ & rig & Mode & & & & & & & & & & & & & & \\
\hline
\end{tabular}

Quelle: SOEP 1984-1999, DJI Regionaldatenbank, Statistik regional 1999, eigene Berechnungen 